\title{
潮滩动力过程影响下扩散边界层和沉积物-水界面 扩散通量的变化
}

\author{
汪嘉宁 ${ }^{1}$ ，赵亮 ${ }^{(1)}$, 魏皓 ${ }^{(2 *}$ \\ (1) 中国海洋大学海洋环境学院, 物理海洋教育部重点实验室, 青岛 266100; \\ (2) 天津科技大学海洋科学与工程学院, 天津 300457 \\ * 联系人, E-mail: weihao@ouc.edu.cn
}

2011-11-18 收稿, 2012-01-06 接受

国家重点基础研究发展计划(2011CB403606)、国家自然科学基金重点项目(40830854)和国家自然科学基金青年科学基金(40806001)资助

\begin{abstract}
摘要 扩散边界层(DBL)控制了沉积物和上覆水间物质的交换, 在潮流底边界层(BBL)的动力 过程影响下, 海洋 DBL 的厚度变化很大, 溶解物质的扩散通量也有很大变化. 因此探究影响 DBL 厚度和扩散通量变化的动力机制是准确估算扩散通量的关键前提，对近海水体营养盐补 充和环境二次污染等问题的准确估计有重要意义. 利用微尺度剖面仪和高频流速仪等对 BBL 的动力结构和 DBL 进行了联合观测. 运用线性分布、剖面拐点和浓度方差法较准确地判识了 DBL 厚度. 分别从水体和沉积物一侧计算了扩散通量, 两者相差不超过 6\%. 使用 PROFILE 模 式对溶解氧剖面进行了精确模拟, 获得了分层的单位体积耗氧速率。结果显示 DBL 厚度 (0.10 0.35 mm) 和扩散通量(15.4 53.6 mmol m $\left.\mathrm{m}^{-2} \mathrm{~d}^{-1}\right)$ 在一个潮周期内均变化了 3.5 倍. 通过进一 步分析动力强迫对 DBL 厚度和扩散通量的影响, 发现在平均流速控制 DBL 厚度占优的潮滩系 统中, $\mathrm{DBL}$ 厚度 $\delta_{\mathrm{DBL}}$ 和平均流速大小 $U$ 成反比, 通过拟合得到了两者的关系式 $\delta_{\mathrm{DBL}}=$ $1686.1 D U^{-1}+0.1$ (其中 $D$ 为分子扩散系数). 扩散通量的变化分阶段受到了沉积物上覆海水和湍 流混合强度变化的影响. 在主要受湍流混合影响的阶段, 扩散通量和湍动能耗散率、摩擦速度 和湍动能的变化都有一定的正相关关系.
\end{abstract}

\section{关键词}

扩散边界层

扩散通量

动力过程

溶解氧

微尺度剖面仪

潮滩 扩散边界层(diffusion boundary layer, DBL) 是沉 积物上很薄的一层, 一般小于 $1 \mathrm{~mm}$, 物质从潮流底 边界层 (bottom boundary layer, BBL)输运到 DBL 后, 分子扩散取代湍扩散成为控制垂直输运的主要因 素 ${ }^{[1 \sim 3]}$. 沉积物和水体的物质交换对海洋中一系列生 物化学过程有着重要作用, 但 DBL 限制了这一交换, 成为沉积物-水界面(sediment-water interface, SWI)扩 散通量的瓶颈. 研究证实 BBL 的动力强迫会显著影 响 DBL 的厚度和 SWI 处溶质的扩散通量(以下简称 扩散通量 $)^{[4 \sim 6]}$. 在 BBL 的动力过程影响下, 海洋 DBL 厚度变化很大, 因而扩散通量也变化很大. 因 此探究影响 DBL 厚度和扩散通量变化的动力机制是
准确估算海底扩散通量的关键前提, 是在水质模型 中建立扩散通量和 BBL 动力情况参数化方案的基础, 对近海水体营养盐补充和环境二次污染等问题的研 究有重要意义. 本文选择生态系统中关键的生源要 素溶解氧作为研究溶质, 其扩散通量的大小对底栖 生物多样性和沉积物中有机物矿化等生物化学过程 有重要作用 ${ }^{[7,8]}$.

流体力学界已有对 DBL 的研究, 张兆顺等人 ${ }^{[9]}$ 对其进行了论述. 流体力学通过对雷诺方程进行量 纲分析, 将靠近壁面的等切应力层分为黏性底层和 对数层, 黏性底层为分子黏性力控制的很薄一层, 即 DBL，对数层为雷诺应力控制的一层, 即 BBL. 海洋

英文版见: Wang J N, Zhao L, Wei H. Variable diffusion boundary layer and diffusion flux at sediment-water interface in response to dynamic forcing over an intertidal mudflat. Chin Sci Bull, 2012, 57, doi: 10.1007/s11434-012-4988-3 
DBL 的系统研究由 Jørgensen 和 Revsbech ${ }^{[1]}$ 首先在实 验室进行, 其利用溶解氧微电极对 DBL 结构进行了 观测(垂直步进精度 0.05 0.1 mm), 发现 DBL 厚度在 0.2 >1 $\mathrm{mm}$ 之间并受到沉积物上覆水流速大小和海 底粗楉度的影响. 随后很多学者对固-液界面上 BBL 和 DBL 并存现象进行了室内研究, 获得了较丰富的 认识. 室内实验发现海底的微地形可以影响溶质在 DBL 的输运, 进而影响 DBL 的结构 ${ }^{[10,11]}$; 在不同物 理图景下 BBL 流速的大小和 DBL 的厚度成反比 ${ }^{[12 ~ 14]}$, 物质输运系数和摩擦速度存在线性关系 ${ }^{[13,14]}$. 除了 室内实验, 自然条件的现场调查也是研究 DBL 的重 要手段. 现场观测主要通过 $1 \mathrm{~mm}$ 内某种物质剖面的 拟合来确定 DBL 的厚度, 并计算扩散通量, 但难点 在于如何获得 $1 \mathrm{~mm}$ 内物质剖面高空间分辨率的变化, 常规观测无法解决这个问题. 借鉴室内实验经验, 通 过观测高空间分辨率溶解氧剖面的方法逐步实现. Reimers 等人 ${ }^{[15]}$ 首先利用溶解氧微电极在东北太平 洋 1000 3000 m 深海对 DBL 进行了现场观测, 垂直 分辨率为 $1 \mathrm{~mm}$, 现在来看这远不能满足对 DBL 的高 分辨率观测研究, 但也显著提高了界面扩散通量的 计算准确度. 随着观测技术的发展, DBL 现场观测的 垂直分辨率由 $0.5 \mathrm{~mm}^{[16]}$ 提高到 $0.05 \mathrm{~mm}^{[2]}$, 基本满足 了对 DBL 的研究. Glud 等人 ${ }^{[17]}$ 对 DBL 结构和扩散 通量进行了观测研究, 系统分析了其时空变化特征. 上述实验室和现场研究均强调了 $\mathrm{BBL}$ 动力过程对
DBL 厚度的重要影响, 但尚未给出 DBL 厚度及扩散 通量与水体的动力学结构的定量关系. Lorke 等人 ${ }^{[18]}$ 和 Bryant 等人 ${ }^{[8]}$ 率先在湖泊中对 BBL 中的动力强迫 和 DBL 进行了综合现场观测，探讨了两者的相关关 系. 但湖泊的动力状况 (一般仅受风的影响)非常弱, 与海洋中的情况差别较大. 据作者所知, 迄今为止还 没有在海洋中联合两者的现场观测研究.

本研究利用 BBL 海床基观测平台和微尺度剖面 仪, 在青岛会场湾潮滩同时进行了 $\mathrm{BBL}$ 动力特征和 DBL 的观测, 推算了溶解氧在 SWI 处扩散通量, 并 对三者的关系进行讨论, 为近海沉积物-水界面物质 扩散与交换机理研究进行了探索和准备.

\section{1 现场观测实施方案}

作者于 2010 年秋在青岛会场湾(图 1)淤泥底质的 潮滩上进行了 BBL 和 DBL 观测. 会场湾的潮汐类型 为半日潮, 受潮汐周期性涨落的影响, 潮滩区域的沉 积物大约只有半个潮周期被海水覆盖, 因此只在 2010 年 11 月 27 日 17 时 20 分到 22 时 40 分期间进 行了有效观测. 将 1 台 Nortek $6 \mathrm{MHz}$ Vector 声学 Doppler 流速仪(ADV)和 1 台 RBR XR-620 温盐深仪 (CTD)放置在 1 个三脚架的海床基平台上进行 BBL 观测. 1 台微尺度剖面仪(自带三脚架)(图 2)放置在海 床基平台附近进行 DBL 同步测量. BBL 和 DBL 观测 平台相距 $1 \mathrm{~m}$, 两者具有基本一致的动力和底质特征.

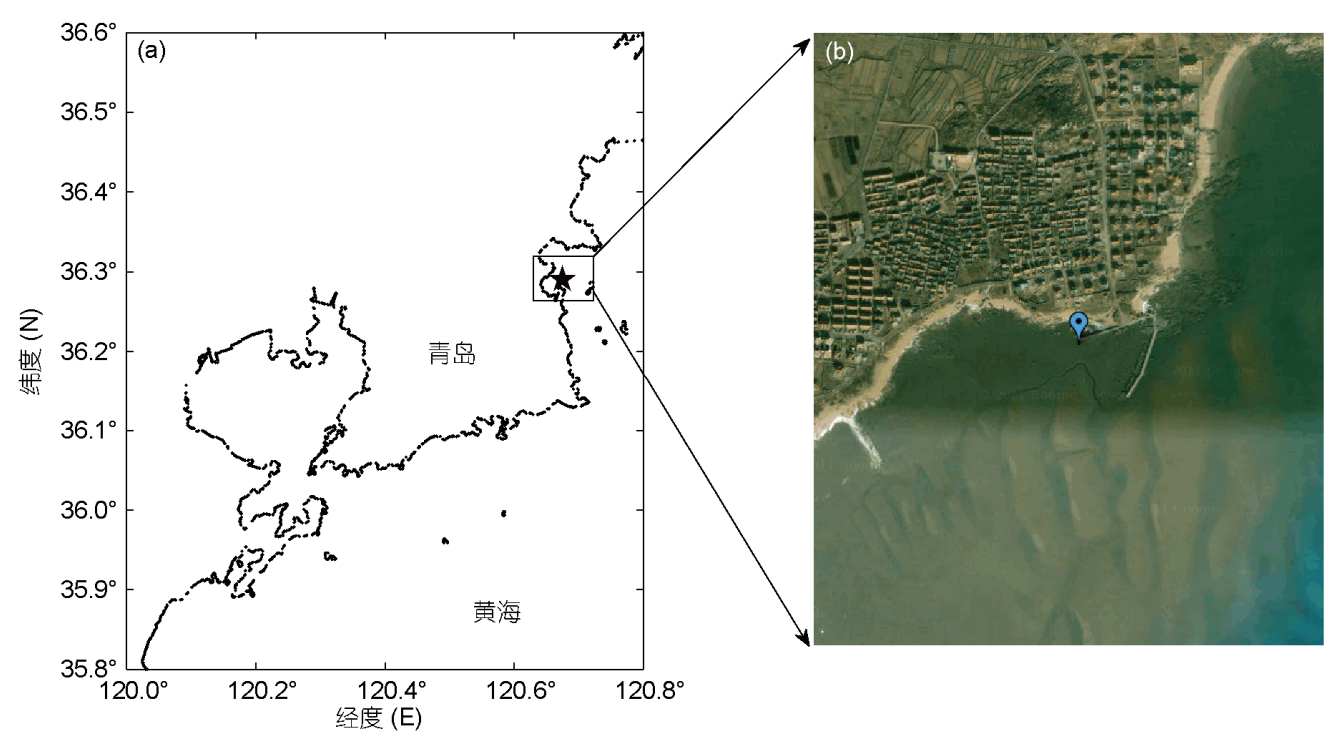

图 1 观测地点青岛会场湾潮滩

观测站位于黑色五角星 (a)和蓝色标识点 (b)的位置, 经纬度: $36.295^{\circ} \mathrm{N}, 120.652^{\circ} \mathrm{E}$, (b)引自 Google Earth 


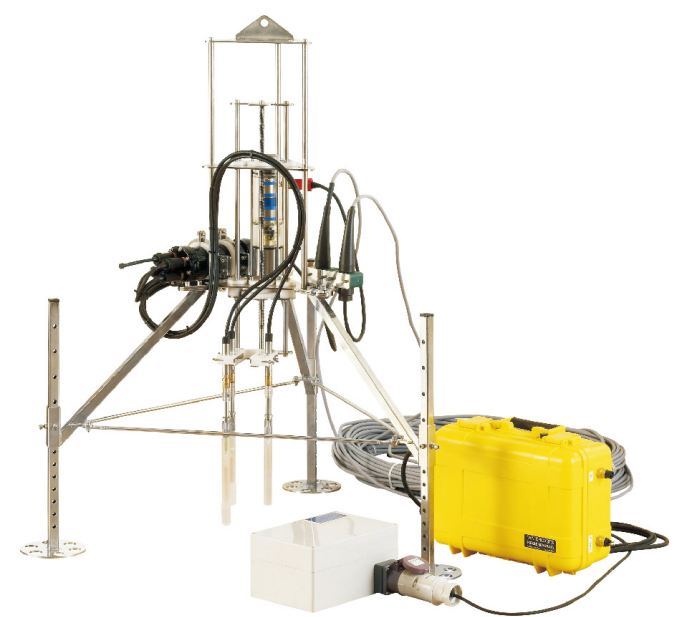

图 2 微尺度剖面仪 MP4

http://www.unisense.com/Default.aspx?ID=97

\section{1 溶解氧微尺度剖面的观测}

微尺度剖面仪(Mini Profiler MP4)主要装备有 1 个 Clark 型溶解氧微电极 $(\mathrm{OX} 25$, Unisense S/A, Denmark)和 1 台微步进控制马达(图 2). 溶解氧微电极的 尖端直径为 $25 \mu \mathrm{m}, 90 \%$ 的响应时间小于 $4 \mathrm{~s}$, 马达垂 直分辨率可达 $50 \mu \mathrm{m}$, 这些保证了对 DBL(厚度小于 1 $\mathrm{mm}$ )微结构的空间高分辨率观测. 电极信号和马达 通过电缆实时由电脑进行存储和控制.

溶解氧浓度剖面每 30 分钟左右观测一次，每个 剖面的测量时间为 $25 \sim 30 \mathrm{~min}$, 间隔 $50 \mu \mathrm{m}$ 取一个点, 每个点重复测量 5 次. 剖面开始于沉积物上 1 2 $\mathrm{mm}$
处，当电极进人沉积物并且信号降到持续的低值即 完成测量, 剖面涵盖了 $\mathrm{BBL}$ 的下边界、 DBL、沉积 物中的整个含氧区(oxic zone)和无氧区(anoxic zone) 的上边界(图 3), 这是推算 DBL 厚度和计算扩散通量 的重要依据.

\section{$1.2 \mathrm{BBL}$ 中动力特征的观测}

1 台 $\mathrm{ADV}$ 固定于海床基平台的中心，采样体积 位于 $0.22 \mathrm{mab}$ (距底高度), 向下进行观测, 采用地球 坐标系(ENU)模式记录数据，以 burst 方式工作，每 10 分钟测量一次, 持续 $6 \mathrm{~min}$, 采样频率为 $8 \mathrm{~Hz}$. Liu 和 $\mathrm{Wei}^{[19]}$ 详细说明了利用 $\mathrm{ADV}$ 资料计算平均流速, 采用惯性耗散法计算湍动能耗散率，采用湍流脉动 相关法计算摩擦速度的方法. (单位质量的)湍动能是 脉动运动所携带的动能, 可用公式 $K=\left(\left(u^{\prime}\right)^{2}+\left(v^{\prime}\right)^{2}+\right.$ $\left.\left(w^{\prime}\right)^{2}\right) / 2$ 求得, 其中 $u^{\prime}, v^{\prime}, w^{\prime}$ 是东向、北向和垂直流速 的脉动量. 我们参考上述文献和公式获得了平均流 速 $(U)$ 、湍动能耗散率 $(\varepsilon)$ 、摩擦速度 $(u *)$ 和湍动能 $(K)$ 等 BBL 中动力特征的值. 1 台 CTD 固定在海床基平 台 $0.40 \mathrm{mab}$ 处，采样频率为 $1 \mathrm{~Hz}$, 对 $\mathrm{BBL}$ 温盐的变 化进行连续观测, 为分子扩散系数的计算提供背景 温盐值.

\section{2 数据处理}

\subsection{DBL 厚度的推算方法}

溶解氧剖面的处理关键是准确定位 SWI 位置(即

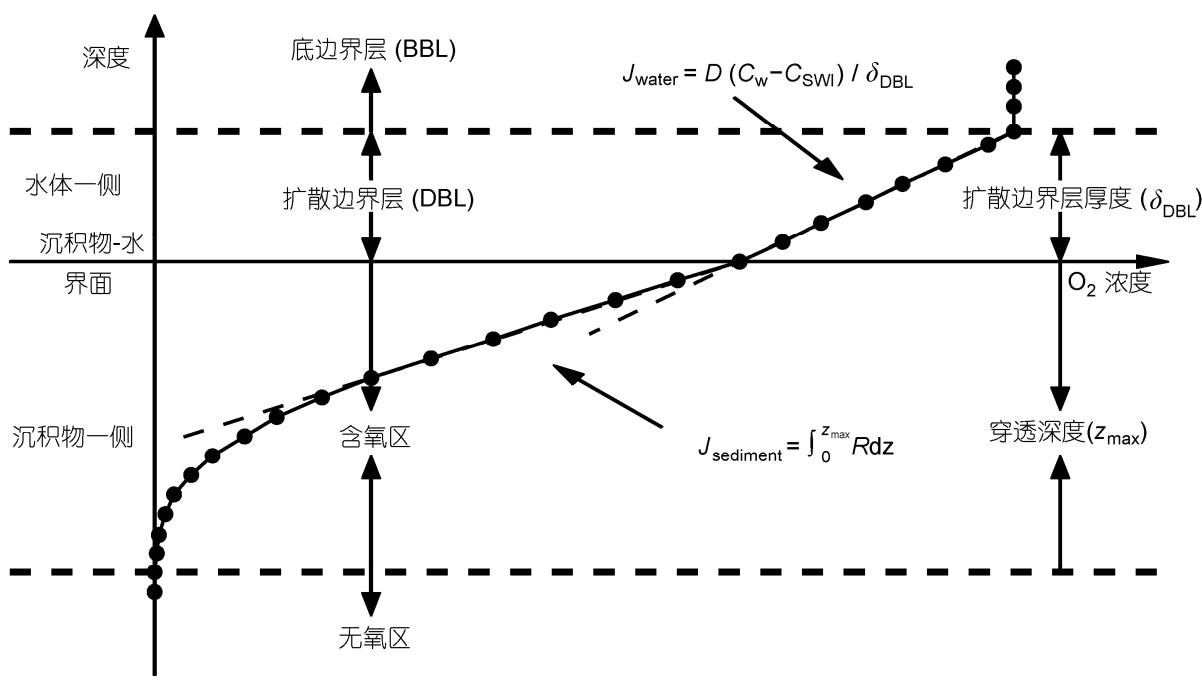

图 3 溶解氧浓度在 BBL, DBL 和沉积物中的理论剖面图 
准确定义 DBL 厚度). 因受到生物扰动和水中气泡等 影响, 溶解氧浓度的分布存在偶然因素, 单纯使用一 种办法往往无法准确估计 DBL 的厚度. 因此本文采 用了溶解氧浓度线性分布法、剖面拐点法和浓度方差 法进行综合判断.

DBL 中的物质输运主要由分子扩散作用控制, 若 DBL 中没有溶解氧的额外消耗和生成, 其垂直浓 度梯度 $\partial C / \partial z$ 应为常数, 溶解氧浓度应从 DBL 上边界 线性减小到 $\mathrm{SWI}^{[14]}$. 因此可以根据剖面的线性分布 (图 3)来判断 DBL 厚度.

如果将 SWI 看成非常薄的一层, 无论从 SWI 的 上方还是下方使用 Fick 第一定律计算扩散通量应该 是相等的, 但 DBL 中分子扩散率是大于沉积物中的, 因此 SWI下方的溶解氧浓度梯度比 SWI 上方的大 ${ }^{[20]}$, 即可以根据溶解氧剖面中线性分布的拐点(即 SWI 处 溶解氧浓度测量点)(图 3)来定义 DBL 厚度。

Lorke 等人 ${ }^{[18]}$ 提出当接近 SWI 时, 溶质浓度波动 减小, 标准方差会随之减小 ${ }^{[2]}$, 因此 SWI 应该在浓度 方差明显减小的地方, 这也是一种判断 DBL 厚度的 方法.

\subsection{SWI 处的溶解氧扩散通量计算方法}

扩散通量也称为沉积物氧气分子扩散吸收量 (diffusion $\mathrm{O}_{2}$ uptake). 对分子扩散吸收量没有影响的 因子, 如沉积物地形的变化、孔隙水的对流和冲洗作 用等, 不在考虑范围内. 扩散通量可以从水体一侧计 算, 也可从沉积物一侧计算(图 3). 本文水体一侧的计 算是根据 Fick 第一定律, 扩散通量 $\left(J_{\text {water }}\right)$ 为

$$
J_{\text {water }}=D \frac{C_{\mathrm{w}}-C_{\mathrm{SWI}}}{\delta_{\mathrm{DBL}}},
$$

其中 $C_{\mathrm{w}}$ 和 $C_{\mathrm{SWI}}$ 为 $\mathrm{BBL}$ 水体中和 $\mathrm{SWI}$ 处的溶解氧浓 度, $\delta_{\mathrm{DBL}}$ 为 $\mathrm{DBL}$ 的厚度, $D$ 为溶解氧分子扩散系数.

本文沉积物一侧的计算是对沉积物中单位体积 耗氧速率进行垂直积分, 扩散通量 $\left(J_{\text {sediment }}\right)$ 为

$$
J_{\text {sediment }}=\int_{0}^{z_{\max }} R \mathrm{~d} z,
$$

其中 $R$ 是 PROFILE模式输出的单位体积耗氧速率(详 见 2.3), $z_{\max }$ 为氧气在沉积物中的穿透深度, $z_{\max }$ 定义 为从 SWI 到溶解氧浓度变为零处的厚度 (图 3).

上述两种办法是都忽略了溶解氧浓度随时间的 变化 $(\partial C / \partial t=0)$. 水体一侧的计算是通常采用的办法, 但在确定 DBL 厚度上存在一定误差. 沉积物一侧的
计算虽然不存在确定 DBL 厚度的问题, 但是对沉积 物中生物化学过程比较敏感, 孔隙度的变化等会引 起误差.

\section{3 溶解氧剖面的模式模拟}

溶解氧观测剖面使用 Berg 等人 ${ }^{[21]}$ 提出的 PROFILE 模式进行数值模拟, 运用模式拟合而不是 简单的将实测数据连接起来可以分辨水中气泡、生物 活动等偶然因素引起的非正常数据, 从而较精确地 体现剖面结构; 同时还可以深化对 DBL 和沉积物中 地球化学过程的研究. 模式使用实测数据作为边界 条件, 采用错误尝试法调节孔隙度等参数使模拟剖 面和实测剖面达到最大拟合度. 模式将沉积物进行 分层, 通过 $F$ 检验得到最佳的分层方式, 进而计算出 每层的单位体积耗氧速率.

PROFILE 模式假设在恒定状态 $(\partial C / \partial t=0)$ 下, DBL 和沉积物中的物质运动由垂直扩散控制, 垂直扩散 主要由 3 种因素引起: 分子扩散作用、生物扰动和冲 洗作用(管居动物的抽吸运动). 体现上述 3 种作用的 一维物质守恒方程为

$$
\frac{\mathrm{d}}{\mathrm{d} x}\left(\varphi\left(D_{\mathrm{s}}+D_{\mathrm{B}}\right) \frac{\mathrm{d} C_{\mathrm{s}}}{\mathrm{d} x}\right)+\alpha\left(C_{\mathrm{w}}-C_{\mathrm{s}}\right)+R=0,
$$

其中 $x$ 为深度, $C_{\mathrm{s}}$ 为沉积物孔隙水的溶质浓度, $C_{\mathrm{w}}$ 为 沉积物上覆海水的溶质浓度, $\varphi$ 为孔隙度, $D_{\mathrm{s}}$ 为在沉 积物中由弯曲度修正的分子扩散率, $D_{\mathrm{B}}$ 为生物扩散 系数, $\alpha$ 为冲洗作用系数, $R$ 为沉积物中单位体积氧气 消耗率或生成率.

为与扩散通量的计算保持一致, 本文忽略生物 扰动和冲洗作用, 即 $\alpha$ 和 $D_{\mathrm{B}}$ 设为零. 剖面的上边界 条件选为 DBL 最上面一点的溶解氧浓度, 下边界条 件选为沉积物含氧区最下面一点的扩散通量, 将其 设置为零. 通过模式模拟沉积物-水界面附近的溶解 氧浓度, 并计算溶解氧扩散通量, 该方法可以有效消 除偶然因素的影响.

\section{3 数据分析}

\subsection{DBL 的厚度和 SWI 处的溶解氧扩散通量}

图 4 是 11 个溶解氧浓度在 BBL, DBL 和沉积物 中的现场观测剖面图. 模式结果和实测值的相关系 数的平方均在 0.99 以上, 说明模式能客观地描述溶 解氧在 DBL 和沉积物中的分布, 恒定状态的假设基 

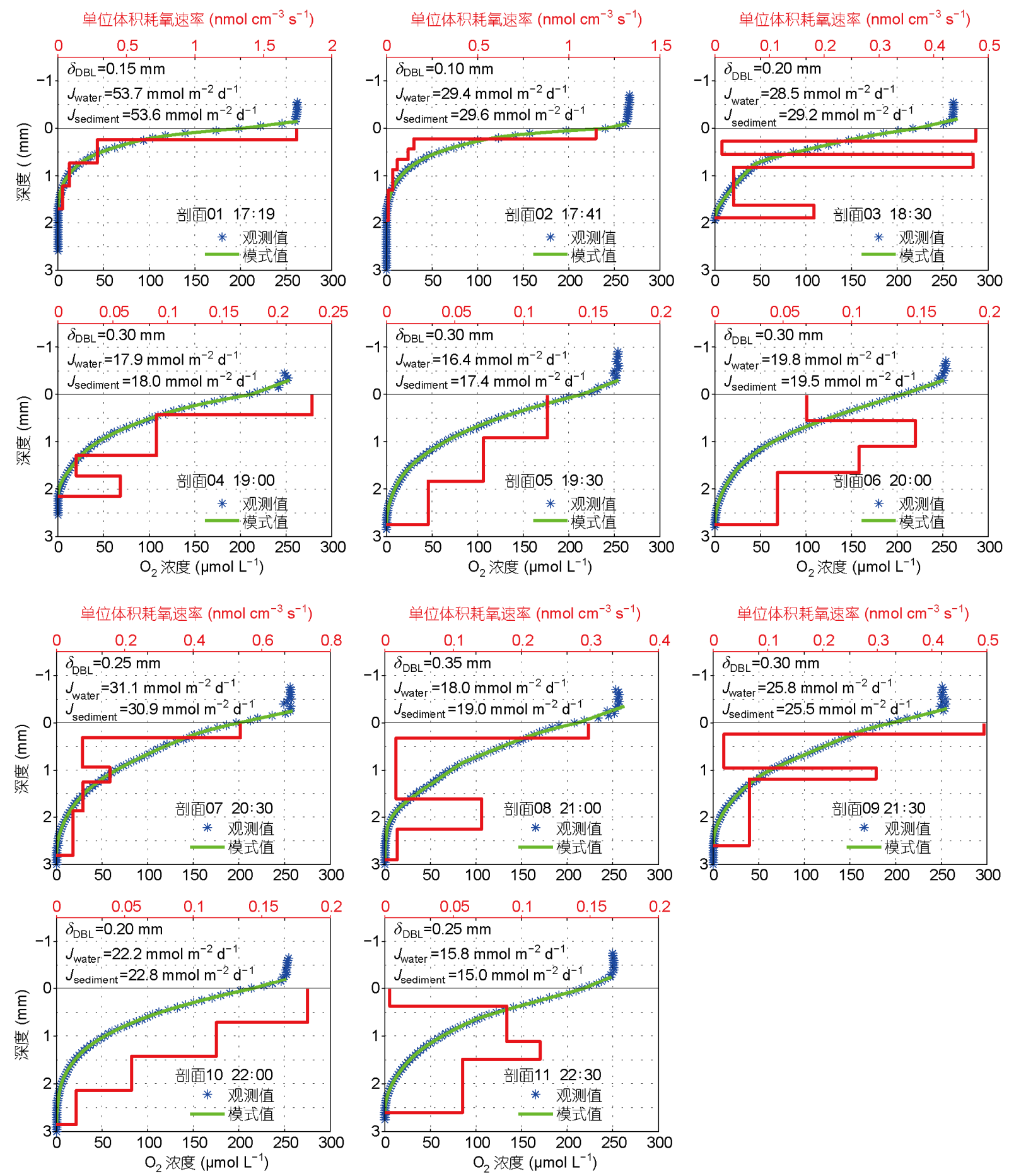

图 4 溶解氧浓度在 BBL, DBL 和沉积物中的现场观测剖面图

蓝色星号表示实测值, 绿线表示 PROFILE 模式的模拟值, 红线表示 PROFILE 模式计算出的沉积物中单位体积耗氧速率, 纵坐标深度 0 表示 SWI, 向下即沉积物一侧为正值

本合理, 生物活动和水中气泡等对剖面的扰动较小, 分子扩散是物质垂直扩散作用的主要因素. 从图上 可以看出溶解氧浓度剖面在 DBL 中是线性分布的,
沉积物中的溶解氧浓度梯度比 DBL 中的大, 浓度梯 度在 SWI 处存在拐点. DBL 厚度在 $0.10 \sim 0.35 \mathrm{~mm}$ 之 间变化, 一个潮周期内改变了 3.5 倍. DBL 平均厚度 
为 $0.25 \mathrm{~mm}$, 说明其是 SWI 上非常薄的一层. 溶解氧 的分子扩散系数 $D$ 由 CTD 所测的温度和盐度来确 定 ${ }^{[22]}$, 其值在 $1.47 \times 10^{-5} \sim 1.51 \times 10^{-5} \mathrm{~cm}^{2} \mathrm{~s}^{-1}$ 之间变化. $J_{\text {water }}$ 和 $J_{\text {sediment }}$ 的相差 $\left(\left(J_{\text {water }}-J_{\text {sediment }}\right) / J_{\text {water }} \times 100 \%\right)$ 不 超过 $6 \%$, 本文将两者进行平均作为每个剖面的扩散 通量. 扩散通量在 $15.4 \sim 53.6 \mathrm{mmol} \mathrm{m} \mathrm{m}^{-2} \mathrm{~d}^{-1}$ 之间变化, 一个潮周期内也改变了 3.5 倍, 平均扩散通量为 25.4 $\mathrm{mmol} \mathrm{m} \mathrm{m}^{-2} \mathrm{~d}^{-1}$.

图 4 还说明当扩散通量增加时, 沉积物中的单位 体积耗氧速率随之增加, 反之亦然. 例如剖面 01(17:19) 的扩散通量为 $53.6 \mathrm{mmol} \mathrm{m}^{-2} \mathrm{~d}^{-1}$, 最大耗氧速率可达 $1.7 \mathrm{nmol} \mathrm{cm}^{-3} \mathrm{~s}^{-1}$, 而剖面 11(22:30)扩散通量为 15.4 $\mathrm{mmol} \mathrm{m}{ }^{-2} \mathrm{~d}^{-1}$, 最大耗氧速率仅为 $0.1 \mathrm{nmol} \mathrm{cm}^{-3} \mathrm{~s}^{-1}$.

图 5 表明沉积物含氧区中溶解氧消耗量并不是 垂直均匀分布的，沉积物含氧区上层 $25 \%$ 的区域消 耗了 $60.5 \pm 13.8 \%$ 的氧气, 这一区域的有氧代谢活动 十分活跃. 而下层 $25 \%$ 的区域仅消耗了 $5.1 \pm 1.8 \%$ 的 氧气(即沉积物含氧区上层 $75 \%$ 的区域中溶解氧累计 消耗后剩余的部分), 说明该区域的有氧代谢活动已 十分微弱. 溶解氧在沉积物表层 $(0 \sim 1 \mathrm{~mm})$ 平均消耗 了 $41.0 \%$, 在 $1 \sim 2 \mathrm{~mm}$ 和 $2 \sim 3 \mathrm{~mm}$ 平均消耗了 $15.0 \%$ 和 $2.0 \%$.

\subsection{BBL 动力强迫和 DBL 厚度、扩散通量的关系}

图 6(a) (d)给出了 BBL 动力特征的时间变化. 湍 动能耗散率、摩擦速度和湍动能具有基本一致的变化
规律, 但平均流速和上述 3 个物理量的对应关系并不 明显, 说明该处湍流并不是单纯由平均流剪切生成 的. 潮滩水深很浅, 物理过程复杂多变, 湍流的产生 可能受到表面波浪、局地地形、泥沙输移和浮力通量 等的影响 ${ }^{[23 \sim 25]}$, 受观测数据的限制且非本文讨论的 重点, 我们不再对这一问题进行深人论述.

图 7(a) (c)显示当平均流速增强时, BBL 中溶解 氧浓度变大, 反之亦然. 剖面 01 04(图 7(a))沉积物中 的溶解氧浓度存在大幅增加的现象, 例如 SWI下方 0.5 $\mathrm{mm}$ 处浓度从 $45.5 \mathrm{umol} \mathrm{L} \mathrm{L}^{-1}$ 增加到 $102.9 \mathrm{umol} \mathrm{L} \mathrm{L}^{-1}$. 这一 过程是由于潮滩(潮间带)沉积物受潮汐的影响出现周 期性的暴露和淹没所产生的, 测量前因为暴露在空气 中沉积物中的间歇水较少, 溶解态的氧含量也相应较 小, 涨潮后沉积物被海水覆盖, 沉积物间隙中的氧气快 速溶于海水, 溶解氧浓度大幅增加. 溶解氧在沉积物中 的穿透深度 $z_{\max }$ 也受到上述过程的影响, 海水中的溶解 氧通过 DBL 的分子扩散作用不断向沉积物输运, 穿透 深度 $z_{\max }$ 在持续增加. 剖面 01 04(图 7(a))的穿透深度 从 $1.75 \mathrm{~mm}$ 快速增加到 $2.20 \mathrm{~mm}$, 但整体来看平均值仅 为 $1.93 \mathrm{~mm}$, 剖面 05 10(图 7 (b), (c)) 的平均穿透深度增 加到 $2.89 \mathrm{~mm}$, 剖面 11(图 7(c))在观测时潮位线位于测 量点附近，溶解氧的扩散作用不能贯穿整个测量时间， 穿透深度减小为 $2.65 \mathrm{~mm}$.

图 6(a)和(e)说明 DBL 厚度和平均流速的大小有 较好的对应关系, 两者的变化成反比, 当平均流速增 加时, DBL 厚度减小, 反之亦然. 例如, 剖面 02(17:41)

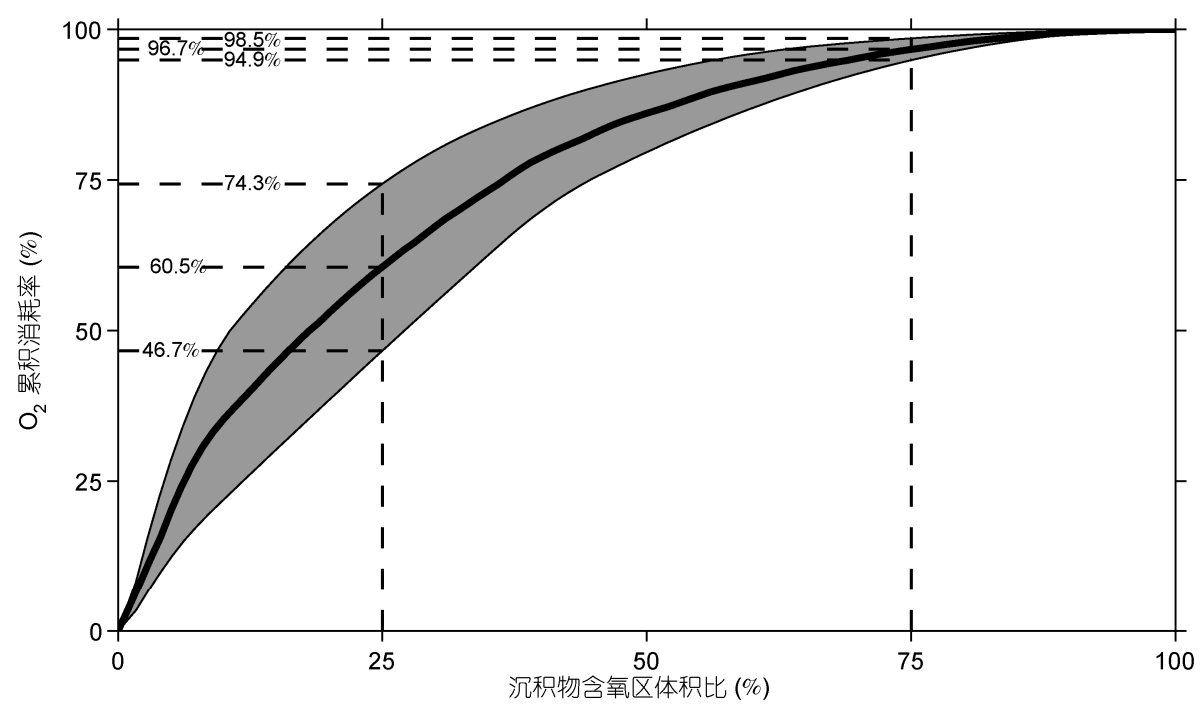

图 5 沉积物含氧区中溶解氧累积消耗率的分布图

阴影部分是根据 11 个剖面计算的溶解氧累积消耗率的分布范围, 阴影中的黑色实线是平均值 


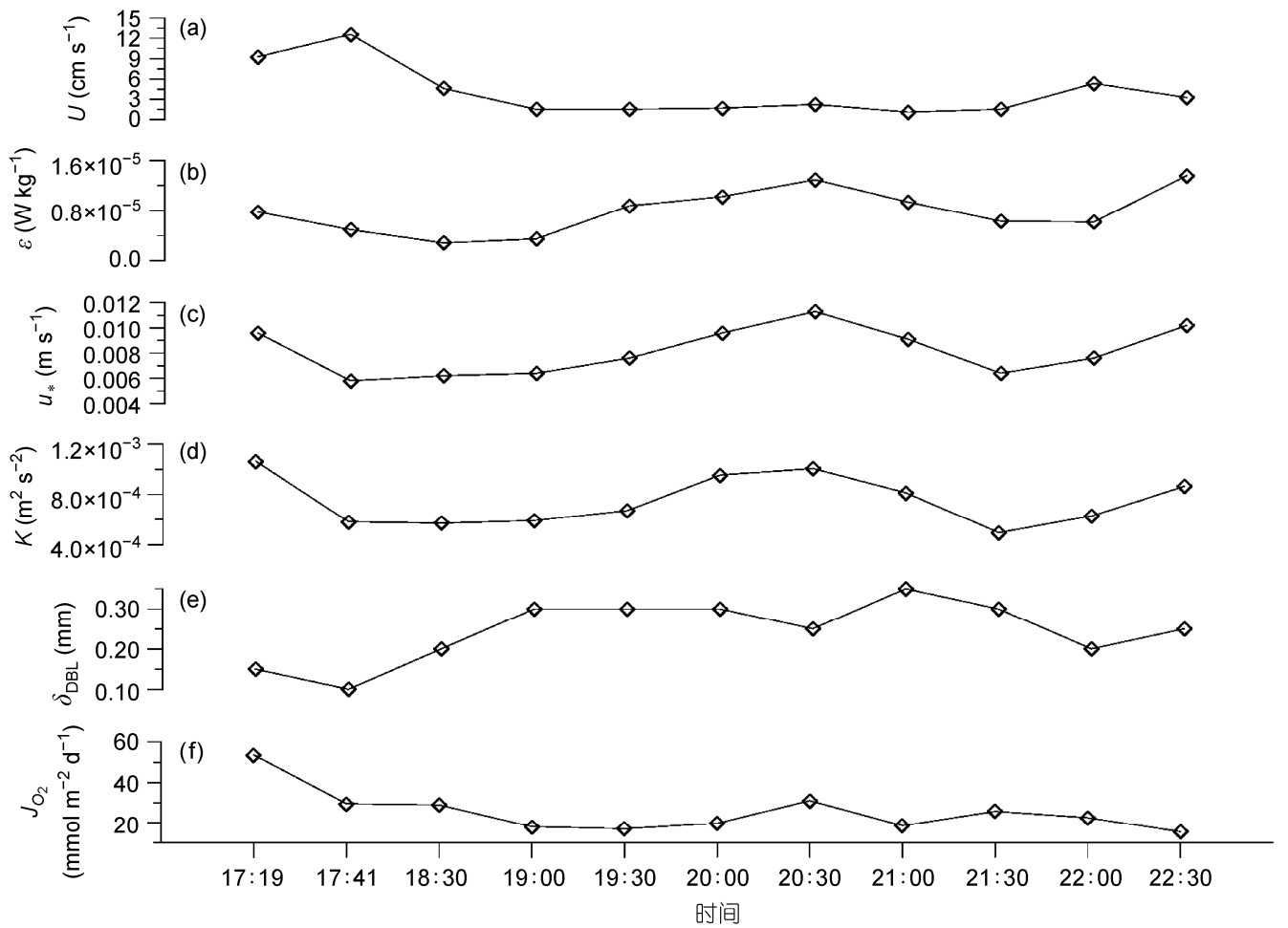

图 $6 \mathrm{BBL}(0.22 \mathrm{mab})$ 的(a)平均流速 $U$, (b) 湍动能耗散率 $\varepsilon 、(\mathrm{c})$ 摩擦速度 $u_{*} 、($ d $)$ 湍动能 $K$ 和(e) DBL 的厚度 $\delta_{\mathrm{DBL}}$ 和(f) SWI 处的扩散通量 $\boldsymbol{J}_{\mathrm{O}_{2}}$ 的时间序列

的平均流速达到最大值 $12.6 \mathrm{~cm} \mathrm{~s}^{-1}$, DBL 厚度减小为 最小值 $0.10 \mathrm{~mm}$. 而剖面 08(21:00)的平均流速减小为 最小值 $1.1 \mathrm{~cm} \mathrm{~s}^{-1}$, DBL 厚度增加到最大值 $0.35 \mathrm{~mm}$. Lorke 等人 ${ }^{[18]}$ 在瑞士 Lucerne 湖的观测发现 DBL 厚度 和湍动能耗散率有很好的对应关系，两者成反比，文 章认为在高能量系统中, 例如河口和潮汐水道, BBL 中平均流速大小控制了 DBL 的厚度; 在低能量系统 中, 例如湖泊和水库, BBL 的湍流强弱控制了 DBL 的 厚度. 这一观点和我们的观测相符, 潮滩作为高能量 系统(湍动能耗散率达 $10^{-6} \mathrm{~W} \mathrm{~kg}^{-1}$ ), $\mathrm{BBL}$ 平均流速大 小而不是湍流强弱控制了 DBL 的厚度. 作者认为从 更本质的角度说, BBL 中平均流速大小和湍流强弱都 可以影响 DBL 的厚度, 平均流会压减或抬升 DBL 厚 度, 湍流会影响溶质浓度脉动的最小尺度来影响 DBL 厚度 (即 Batchelor 尺度 $\mathrm{L}_{\mathrm{B}}=2 \pi\left(v D^{2} / \varepsilon\right)^{1 / 4}$ ), 两者控 制 DBL 厚度的最小值决定了实际厚度.

图 6(a)和(e)已说明在平均流控制 DBL 厚度占优 的系统中, 平均流速的大小和 DBL 的厚度成反比, 下面我们试图将平均流速 $U$ 和物质输运系数 $\beta(\beta=$ $\left.D / \delta_{\mathrm{DBL}}\right)$ 的关系进行量化, 以期在数值模式中找到定
义 DBL 厚度的参数化方案. 根据量纲分析的基本定 理( $\mathrm{Pi}$ 定理), $\mathrm{DBL}$ 厚度 $\delta_{\mathrm{DBL}}$ 和平均流速 $U$ 、分子扩散 系数 $D$ 的关系应该如下: $\delta_{\mathrm{DBL}}=a D U^{-1}+b$, 其中 $a$ 和 $b$ 为参数. 图 8(a) 是 DBL 厚度和 BBL 中 $D U^{-1}$ 关系的散 点图, 对两者进行多项式拟合(黑线), 发现两者有很 好的线性关系 $\delta_{\mathrm{DBL}}=1686.1 D U^{-1}+0.1$, 其中分子扩散 系数 $D$ 的单位为 $\mathrm{mm}^{2} \mathrm{~s}^{-1}$, 平均流速 $U$ 的单位为 $\mathrm{mm} \mathrm{s}^{-1}$, $\mathrm{DBL}$ 厚度 $\delta_{\mathrm{DBL}}$ 的单位为 $\mathrm{mm}$. 图 8(b) 利用这一拟合关 系式计算了 DBL 厚度, 结果显示拟合值和实测值相关 系数的平方 $\left(R^{2}\right)$ 为 0.90 , 线性拟合的标准方差 $(S D)$ 为 0.02 , 说明关系式较好地预测了 $\mathrm{DBL}$ 的厚度.

图 6(b)和(f)分别显示了湍动能耗散率和扩散通 量的时间序列. 除剖面 11 外, 扩散通量的变化规律 可以分为两个阶段(剖面 01 04 和 05 10)来解释, 两 个阶段分别受到了沉积物上覆海水和湍动能耗散率 变化的影响. 剖面 01 04 扩散通量较大, 平均达 37.3 $\mathrm{mmol} \mathrm{m} \mathrm{m}^{-2} \mathrm{~d}^{-1}$, 这是由于在测量前沉积物暴露在空气 中间歇水较少，溶解态的氧含量也较小，涨潮后沉积 物和海水形成较大的溶解氧浓度差引起的, 图 7(a)显 示剖面 01 04 中沉积物溶解氧浓度较低也说明了这 

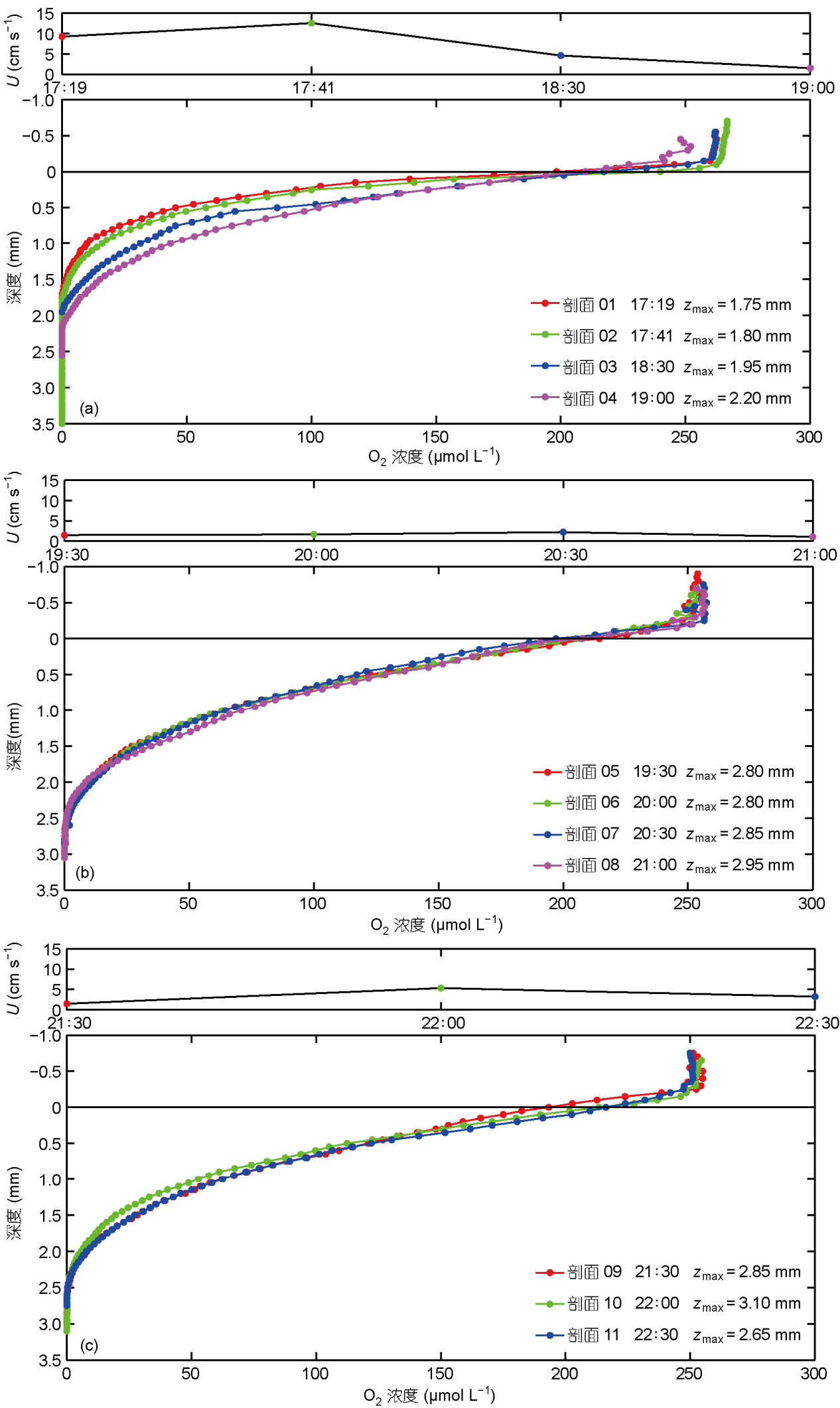

图 7 涨潮(a)、平潮(b)和落潮(c)阶段 BBL 平均流速 $U$ 和溶解氧浓度剖面的关系图 

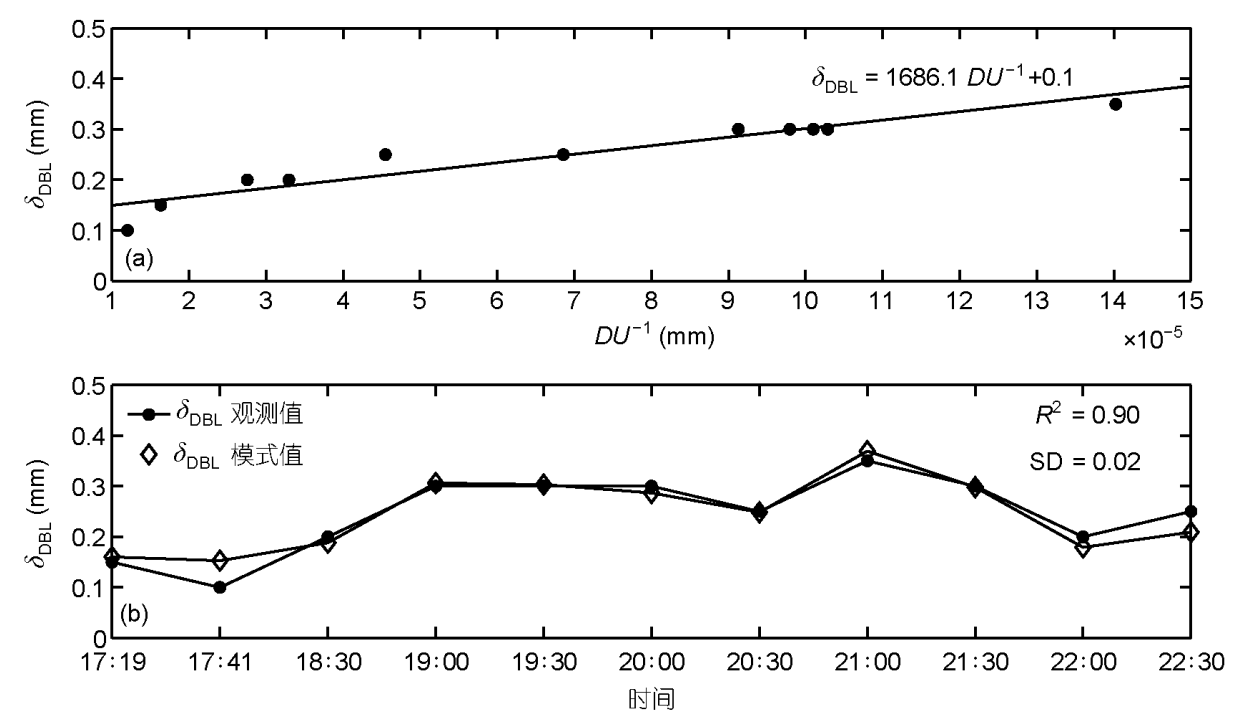

图 8 (a) BBL 中 $D U^{-1}$ 和 DBL 厚度 $\delta_{\mathrm{DBL}}$ 关系的散点拟合图; (b) DBL 厚度 $\delta_{\mathrm{DBL}}$ 的拟合关系式值(菱形)和实测值(实心圆)的 时间序列图

个问题. 剖面 05 10 扩散通量和湍动能耗散率的变化 规律基本一致, 结合图 6(b) (d)说明扩散通量和湍动 能耗散率、摩擦速度和湍动能的变化都有一定的正相 关关系. 当湍动能耗散率(摩擦速度或湍动能)增加时, 湍流混合增强, 推测其通过增加 BBL 中溶解氧的浓 度或是减小 DBL 的厚度来加大扩散通量, 反之亦然. 剖面 11 虽然湍动能耗散率较大, 但潮位线已退至测 量点附近, 溶解氧的扩散作用不能贯穿整个测量时 间，扩散通量减小.

\section{4 讨论与结论}

本文通过实测数据分析了 DBL 和扩散通量 对 BBL 动力强迫的响应, 相关的讨论和重要结论 如下:

运用溶解氧浓度剖面的线性分布、剖面拐点和浓 度方差法可以较准确的判断 SWI 的位置并定义 DBL 的厚度. DBL 的厚度在 $0.10 \sim 0.35 \mathrm{~mm}$ 之间变化, 一个 潮周期内改变了 3.5 倍. DBL 平均厚度为 $0.25 \mathrm{~mm}$, 是 SWI 上非常薄的一层. 扩散通量在 $15.4 \sim 53.6 \mathrm{mmol}$ $\mathrm{m}^{-2} \mathrm{~d}^{-1}$ 之间变化, 一个潮周期内也改变了 3.5 倍. 溶 解氧剖面得到的 DBL 对其他溶质也同样适用, 如 $\mathrm{NO}^{3-[26]}$. DBL 厚度对扩散通量的计算至关重要, 受 观测手段限制传统计算扩散通量时一般将 DBL 厚度 (或浓度梯度的分层)取为厘米量级，而本文现场观测 发现 DBL 厚度仅为毫米量级, 因此在这种情况下传
统计算方法无法获得对扩散通量的准确认识. 因此 对 DBL 的研究是深人了解 SWI 处的营养盐交换通量 和沉积物营养盐再生等的关键性问题.

BBL 的动力强迫可以显著影响 DBL 的厚度和扩 散通量. BBL 中溶解氧浓度和平均流速有正相关关系. 在平均流速控制 DBL 厚度占优的潮滩系统中, DBL 厚度和平均流速大小成反比, 而且两者具有很好的 线性关系 $\delta_{\mathrm{DBL}}=1686.1 D U^{-1}+0.1$, 通过上述关系式计 算的拟合值和实测值的相关系数平方达 0.90 , 这一 关系式是对 Batchelor 尺度 $\left(L_{\mathrm{B}}=2 \pi\left(v D^{2} / \varepsilon\right)^{1 / 4}\right)$ 来定义 DBL 厚度的一个补充 ${ }^{[27]}$, 因为 Batchelor 尺度被证实 可以在湍流混合控制 DBL 厚度占优的系统中较好地 预报 DBL 厚度 ${ }^{[18]}$. 非常有意思的是观测研究发现

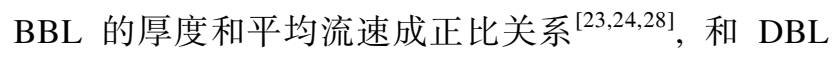
正好相反, 这构建了潮流边界层随宏观流速变化的 物理图景, BBL 和 DBL 的相互联系和影响值得深人 研究. 扩散通量的变化分阶段受到了沉积物上覆海 水和湍流混合强度的影响. 在主要受湍流混合影响 的阶段, 扩散通量和湍动能耗散率、摩擦速度和湍动 能的变化都有一定的正相关关系.

海洋潮滩因受潮汐的影响, 沉积物出现周期性 的暴露和淹没, DBL 厚度和扩散通量变化的动力机 制和开阔海洋会有所不同. 今后我们将在开阔海洋 中进行更长时间序列的观测, 以期更好地探究 BBL 动力强迫下 $\mathrm{DBL}$ 和扩散通量的变化规律. 
1 Jørgensen B B, Revsbech N P. Diffusive boundary layers and the oxygen uptake of sediments and detritus. Limnol Oceanogr, 1985, 30: $111-122$

2 Gundersen J K, Jørgensen B B. Microstructure of diffusive boundary layers and the oxygen uptake of the sea floor. Nature, 1990, 345: 604-607

3 汪亚平, 高抒, 贾建军. 海底边界层水流结构及底移质搬运研究进展. 海洋地质与第四纪地质, 2000, 3: 101-106

4 Higashino M, Stefan H G, Gantzer C J. Periodic diffusional mass transfer near sediment/water interface: Theory. J Environ Eng, 2003, 129: $447-455$

5 Higashino M, Gantzer C J, Stefan H G. Unsteady diffusional mass transfer at the sediment/water interface: Theory and significance for SOD measurement. Water Res, 2004, 38: 1-12

6 Higashino M, O'Connor B L, Hondzo M, et al. Oxygen transfer from flowing water to microbes in an organic sediment bed. Hydrobiologia, 2008, 614: 219-231

7 Berg P, Glud R N, Hume A, et al. Eddy correlation measurements of oxygen uptake in deep ocean sediments. Limnol Oceanogr-Meth, 2009, 7: 576-584

8 Bryant L D, Lorrai C, McGinnis D F, et al. Variable sediment oxygen uptake in response to dynamic forcing. Limnol Oceanogr, 2010, 55: 950-964

9 张兆顺, 崔桂香, 许春晓. 湍流理论与模拟. 北京: 清华大学出版社, 2005. 98-102

10 Jørgensen B B, Des Marais D J. The diffusive boundary layer of sediments: Oxygen microgradients over a microbial mat. Limnol Oceanogr, 1990, 35: 1343-1355

11 Røy H, Hüttel M, Jørgensen B B. The role of small-scale sediment topography for oxygen fluxes across the diffusive boundary layer. Limnol Oceanogr, 2002, 47: 837-847

12 Sweerts J-P R A, Louis V S T, Cappenberg T E. Oxygen concentration profiles and exchange in sediment cores with circulated overlying water. Freshwater Biol, 1989, 21: 401-409

13 Hondzo M. Dissolved oxygen transfer at the sediment-water interface in a turbulent flow. Water Resour Res, 1998, 34: 3525-3533

14 Steinberger N, Hondzo M. Diffusional mass transfer at sediment-water interface. J Environ Eng, 1999, 125: 192-200

15 Reimers C E, Fischer K M, Merewether R, et al. Oxygen microprofiles measured in situ in deep ocean sediments. Nature, 1986, 320: 741-744

16 Archer D, Emerson S, Smith C R. Direct measurement of the diffusive sublayer at the deep sea floor using oxygen microelectrodes. Nature, 1989, 340: 623-626

17 Glud R N, Stahl H, Berg P, et al. In situ microscale variation in distribution and consumption of $\mathrm{O}_{2}$ : A case study from a deep ocean margin sediment (Sagami Bay, Japan). Limnol Oceanogr, 2009, 54: 1-12

18 Lorke A, Müller B, Maerki M, et al. Breathing sediments: The control of diffusive transport across the sediment-water interface by periodic boundary-layer turbulence. Limnol Oceanogr, 2003, 48: 2077-2085

19 Liu Z, Wei H. Estimation to the turbulent kinetic energy dissipation rate and bottom shear stress in the tidal bottom boundary layer of the Yellow Sea. Prog Nat Sci, 2007, 17: 289-297

20 Røy H, Huettel M, Jørgensen B B. Transmission of oxygen concentration fluctuations through the diffusive boundary layer overlying aquatic sediments. Limnol Oceanogr, 2004, 49: 686-692

21 Berg P, Risgaard-Petersen N, Rysgaard S. Interpretation of measured concentration profiles in sediment pore water. Limnol Oceanogr, 1998, 43: $1500-1510$

22 Ramsing N, Gundersen J. Seawater and gases-Tabulated physical parameters of interest to people working with microsensors in marine systems. Version 2.0. Unisense Internal Report. 1994

23 Liu H, Wu C, Xu W, et al. Contrasts between estuarine and river systems in near-bed turbulent flows in the Zhujiang (Pearl River) Estuary, China. Estuar Coast Shelf S, 2009, 83: 591-601

24 Wu J, Liu H, Ren J, et al. Cyclonic spirals in tidally accelerating bottom boundary layers in the Zhujiang (Pearl River) Estuary. J Phys Oceanogr, 2011, 41: 1209-1226

25 汪亚平, 高抒, 贾建军. 浪流联合作用下潮滩沉积动力过程的高分辨率数据采集与分析. 科学通报, 2006, 51: 339-348

26 Lorenzen J, Larsen L H, Kjær T, et al. Biosensor determination of the microscale distribution of nitrate, nitrate assimilation, nitrification and denitrification in a diatom-inhabited freshwater sediment. Appl Environ Microbiol, 1998, 64: 3264-3269

27 Hearn C J, Robson J. Modelling a bottom diurnal boundary layer and its control of massive alga blooms in an estuary. Appl Math Mod, 2000, 24: 843-859

28 Lozovatsky I D, Liu Z, Wei H, et al. Tides and mixing in the northwestern East China Sea Part I: Rotating and reversing flows. Cont Shelf Res, 2008, 28: 318-337 